\title{
A stochastic model of HIV infection incorporating combined therapy of HAART driven by Lévy jumps
}

\author{
Yan Cheng ${ }^{1 *}$, Fumin Zhang ${ }^{2}$ and Min Zhao
}

"Correspondence:

chengy79@163.com

'School of Mathematical, Taiyuan

University of Technology, Taiyuan, China

Full list of author information is available at the end of the article

\begin{abstract}
A stochastic HIV infection model of virus-to-cell transmission is proposed, incorporating the antiretroviral drug therapy by introducing efficacy parameters of RTI and PI drugs, considering the Lévy noise for the inherent stochastic biochemical processes. First, we discuss the model existence of a global positive solution and, by applying Itô's formula, establish a sufficient condition for the extinction of infected $\mathrm{CD}^{+} \mathrm{T}$-cells and virus particles. Then, for proving the persistence in mean, a special method is investigated to handle the model. It is obtained that if $\tilde{R}_{1}>1$ the infected $\mathrm{CD}^{+} \mathrm{T}$-cells and virus particles will be persistent in mean. Finally, some numerical simulations are carried out to show the effects of inherent stochastic fluctuation.
\end{abstract}

Keywords: Stochastic epidemic model; Lévy jump; Antiretroviral drug therapy; Persistence in mean

\section{Introduction}

Human immunodeficiency virus (HIV) began to spread world-wide decades ago. The $\mathrm{CD}^{+}{ }^{+} \mathrm{T}$-cells are the primary targets of HIV infection, when virus particles invade the human immune system [1]. The progress of HIV infection has several different stages, including the early stage of infection, the clinical latency stage, the stage of immune system becoming damaged, and the final stage when HIV progresses to acquired immunodeficiency syndrome (AIDS) - a fatal disease, so far, there is no cure [2].

At the early stage of infection, virus-to-cell transmission is the main route of HIV viral infection in within-host dynamics. When virus invades the human immune system, the immune system can produce antibodies and destroy most of the virus, just the virus concentration declines. But in this stage, patients always show flu-like symptoms, which generally are diagnosed. If medication starts in this stage, the virus concentration is low, random fluctuations may have a significant effect on the dynamics of the disease, there is a probability to prevent the disease development, it can prolong the life expectancy of patients; therefore, therapy in the early stage is of vital importance [3].

Over the last two decades, there has been extensive research on the early stage of HIV infection. Mathematical models describing the interaction between virus and CD4 $4^{+} \mathrm{T}$-cells have been a major area of the research, which plays an important role in analyzing the

(c) The Author(s) 2019. This article is distributed under the terms of the Creative Commons Attribution 4.0 International License (http://creativecommons.org/licenses/by/4.0/), which permits unrestricted use, distribution, and reproduction in any medium, provided you give appropriate credit to the original author(s) and the source, provide a link to the Creative Commons license, and indicate if changes were made. 
behavior of the system on a cellular level to explain different phenomena. Various models have been researched from the perspective of virus-to-cell transmission [4-11].

The general pattern of viral load decay in the early stage of HIV patients is to treat them with antiretroviral therapy and to suppress HIV replication inside the host cell [12]. Mathematical models including antiretroviral therapy have been proposed to study the effects of drug therapy, and they provided theoretical principles to facilitate the development of treatment strategies for HIV infected patients. In Ref. [13], the authors considered the therapy of reverse transcriptase inhibitors (RTIs) drugs which have different drug efficacy on CD4+ T cells, to block conversion of uninfected cells to infected cells. In Refs [14, 15], protease inhibitor (PI) drugs therapy was designed to intervene replication of the virus, make the newly produced virus noninfectious. Currently, evidence showed that highly active antiretroviral therapy (HAART) is one of the most effective ways to suppress virus replication and progression, which is a combination prevention interventions strategy. HAART comprises the effect of reverse transcriptase inhibitor (RTI) drugs and protease inhibitor (PI) drugs. In Refs [16, 17], by establishing mathematical models to describe the effects of combined RTIs and PIs treatments on HIV infection, the result confirmed that PIs drugs are more effective than RTIs drugs, and the combined therapy of RTIs and PIs is more effective than monotherapy of RTIs or PIs.

Mathematical models of HIV infection process traditionally take the form of deterministic differential equations. Various deterministic models have been established in the literature to describe the dynamics of healthy and infected $C D 4^{+} \mathrm{T}$-cells and virus particles. Especially, in Ref. [18], Mao et al. proposed a deterministic model to describe the viral dynamics of HIV-1 infection in the presence of HAART:

$$
\left\{\begin{array}{l}
\frac{\mathrm{d} x_{1}}{\mathrm{~d} t}=\lambda-\delta x_{1}-\left(1-\gamma_{\mathrm{RTI}}\right) \beta x_{1} x_{3}, \\
\frac{\mathrm{d} x_{2}}{\mathrm{~d} t}=\left(1-\gamma_{\mathrm{RTI}}\right) \beta x_{1} x_{3}-a x_{2}, \\
\frac{\mathrm{d} x_{3}}{\mathrm{~d} t}=\left(1-\eta_{\mathrm{PI}}\right) N a x_{2}-\mu x_{3}-\left(1-\gamma_{\mathrm{RTI}}\right) \beta x_{1} x_{3} .
\end{array}\right.
$$

The parameters in the model are as follows:

$x_{1}(t)$ is the concentration of healthy CD4+ $4^{+}$-cells;

$x_{2}(t)$ is the concentration of infected CD4 $4^{+} \mathrm{T}$-cells;

$x_{3}(t)$ is the concentration of free virus particles;

$\lambda$ is the rate at which new target cells are generated per unit time;

$\delta$ is the death rate of healthy cells;

$\beta$ is the transmission coefficient between uninfected cells and infective virus particles;

$a$ is the death rate of infected cells and viral lysis;

$N$ is the number of new particles released by each infected cell when it lyses;

$\mu$ is the rate of virus particles cleared from the system;

$\gamma_{\text {RTI }}$ is the efficacy of reverse transcriptase inhibitor drug effect;

$\eta_{\mathrm{PI}}$ is the efficacy of protease inhibitor drug.

In the presence of antiretroviral drugs therapy, not all cells are able to react to the drugs, so the healthy $\mathrm{CD}_{4}^{+} \mathrm{T}$-cells diminish by infection through contact with the virus at a reduced rate $\beta\left(1-\gamma_{\mathrm{RTI}}\right)$, and $0<\gamma_{\mathrm{RTI}}<1$. Due to the effects of PIs drugs, viral burst size $\mathrm{N}$ is reduced to $N\left(1-\eta_{\mathrm{PI}}\right)$, and $0<\eta_{P} I<1$. When a single infective virus particle infects a single healthy $\mathrm{CD}_{4}^{+} \mathrm{T}$-cell, the virus particle is absorbed into the healthy CD4 ${ }^{+} \mathrm{T}$-cell and effectively dies, hence the term $\beta\left(1-\gamma_{\mathrm{RTI}}\right) x_{1} x_{3}$ appears in all the three equations. 
The basic reproduction number is obtained in Ref. [18]:

$$
R_{0}=\frac{\left(1-\gamma_{\mathrm{RTI}}\right) \beta \lambda N\left(1-\eta_{\mathrm{PI}}\right)}{\delta \mu+\beta \lambda\left(1-\gamma_{\mathrm{RTI}}\right)}
$$

which means the expected number of secondary infected cells caused by a single infected cell entering the disease-free population at equilibrium. If $R_{0}<1$, the model has a diseasefree equilibrium $\left(\frac{\lambda}{\delta_{1}}, 0,0\right)$; when $R_{0}>1$, there is a unique endemic equilibrium given by $\left(x_{1}^{*}, x_{2}^{*}, x_{3}^{*}\right)$, where

$$
\begin{aligned}
& x_{1}^{*}=\frac{\mu}{\beta\left(1-\gamma_{\mathrm{RTI}}\right)\left[N\left(1-\eta_{\mathrm{PI}}\right)-1\right]}, \\
& x_{2}^{*}=\frac{\beta \lambda\left(1-\gamma_{\mathrm{RTI}}\right) N\left(1-\eta_{\mathrm{PI}}\right)-\beta \lambda\left(1-\gamma_{\mathrm{RTI}}\right)-\delta \mu}{\beta \alpha\left(1-\gamma_{\mathrm{RTI}}\right)\left[N\left(1-\eta_{\mathrm{PI}}\right)-1\right]}, \\
& x_{3}^{*}=\frac{\beta \lambda\left(1-\gamma_{\mathrm{RTI}}\right) N\left(1-\eta_{\mathrm{PI}}\right)-\beta \lambda\left(1-\gamma_{\mathrm{RTI}}\right)-\delta \mu}{\beta\left(1-\gamma_{\mathrm{RTI}}\right)\left[\left(1-\gamma_{\mathrm{RTI}}\right) \beta \mu\right]},
\end{aligned}
$$

and the infected cells and infective virus particles will persist to exist (see Ref. [18]).

However, for the process of HIV infection in the early stage, the successful infection may be affected by many factors after a virus particle attaches to a healthy $\mathrm{CD} 4^{+} \mathrm{T}$-cell, such as reverse transcription, nuclear import, and the patient immune system strength. The effect of these factors on the dynamics of early infection is important. Deterministic models are not applicable to describe this random behavior.

It is well recognized that HIV infection is an inherently random process in viral gene production, different $\mathrm{CD} 4^{+} \mathrm{T}$-cells and infective virus particles reacting in the same environment can often give different results [19]. Singh et al. showed that stochastic expression of human immunodeficiency virus HIV proteins can affect the viral fate-decision between active replication and post integration latency in single cells by experimental data [20]. Deterministic models are not applicable to describe the random variations in many biological factors; therefore, it is feasible to consider the random variable in an HIV dynamical model, see Refs [21-25]. In [18] Mao et al. considered the effect of environmental stochasticity on some of the model parameters (1.1), then the stochastic differential equations become:

$$
\left\{\begin{array}{l}
\mathrm{d} x_{1}=\left[\lambda-\delta x_{1}-\left(1-\gamma_{\mathrm{RTI}}\right) \beta x_{1} x_{3}\right] \mathrm{d} t+\sigma_{1} x_{1} \mathrm{~d} B_{1}(t), \\
\mathrm{d} x_{2}=\left[\left(1-\gamma_{\mathrm{RTI}}\right) \beta x_{1} x_{3}-a x_{2}\right] \mathrm{d} t+\sigma_{2} x_{2} \mathrm{~d} B_{2}(t), \\
\mathrm{d} x_{3}=\left[\left(1-\eta_{\mathrm{PI}}\right) N a x_{2}-\mu x_{3}-\left(1-\gamma_{\mathrm{RTI}}\right) \beta x_{1} x_{3}\right] \mathrm{d} t+\sigma_{3} x_{3} \mathrm{~d} B_{3}(t) .
\end{array}\right.
$$

Here, $B_{1}(t)$ and $B_{2}(t)$ are independent standard Brownian motions.

However, due to the inherent stochastic nature of biochemical processes, the dynamic process of HIV viral infection may suffer the strong fluctuation such that the classical stochastic model (1.2) cannot explain the strong, occasional fluctuations of the biological environment. In this work, we propose an extracellular stochastic model to describe the phenomena in the initial stages of HIV infection. The jump diffusion model can describe the phenomena causing a big jump to occur occasionally [26-28]. The stochastic 
differential equations derived by considering the Poisson process are as follows:

$$
\left\{\begin{aligned}
\mathrm{d} x_{1}(t)= & {\left[\lambda-\delta x_{1}-\left(1-\gamma_{\mathrm{RTI}}\right) \beta x_{1} x_{3}\right] \mathrm{d} t+\sigma_{1} x_{1} \mathrm{~d} B_{1}(t) } \\
& +\int_{Y} \gamma_{1}(u) x_{1}(t-) \tilde{N}(\mathrm{~d} t, \mathrm{~d} u), \\
\mathrm{d} x_{2}(t)= & {\left[\left(1-\gamma_{\mathrm{RTI}}\right) \beta x_{1} x_{3}-a x_{2}\right] \mathrm{d} t+\sigma_{2} x_{2} \mathrm{~d} B_{2}(t)+\int_{Y} \gamma_{2}(u) x_{2}(t-) \tilde{N}(\mathrm{~d} t, \mathrm{~d} u), } \\
\mathrm{d} x_{3}(t)= & {\left[\left(1-\eta_{\mathrm{PI}}\right) N a x_{2}-\mu x_{3}-\left(1-\gamma_{\mathrm{RTI}}\right) \beta x_{1} x_{3}\right] \mathrm{d} t+\sigma_{3} x_{3} \mathrm{~d} B_{3}(t) } \\
& +\int_{Y} \gamma_{3}(u) x_{3}(t-) \tilde{N}(\mathrm{~d} t, \mathrm{~d} u) .
\end{aligned}\right.
$$

Here, $x_{1}(t-), x_{2}(t-)$, and $x_{3}(t-)$ are the left limit of $x_{1}(t), x_{2}(t)$, and $x_{3}(t), \tilde{N}(\mathrm{~d} t, \mathrm{~d} u)=$ $N(\mathrm{~d} t, \mathrm{~d} u)-v(\mathrm{~d} u) \mathrm{d} t, N$ is a Poisson counting measure with characteristic measure $v$ on a measurable subset $Y$ of $(0,+\infty)$ with $v(Y)<\infty \cdot \gamma_{i}(u): Y \times \Omega \rightarrow R$ is bounded and continuous $(i=1,2,3)$. We assume that $B_{i}(t)(i=1,2,3)$ and $N$ are independent throughout the paper.

By considering the results in the above references, the main contributions of this paper are as follows:

- Describe the strong fluctuation of HIV viral infection by introducing a Lévy jump process into the HIV viral dynamical model, it can be seen as an extension of [18];

- By using the Hasminskii-Mao theorem and stochastic Lyapunov function, we show that the model has a unique global positive solution;

- In order to investigate the sufficient conditions of infected CD4 $4^{+} \mathrm{T}$-cells and virus particles persistence in mean, we applied a new method to establish a stochastic Lyapunov function.

\section{Preliminaries}

First, we introduce the following notations. Throughout this paper, let $\left(\Omega, \mathcal{F},\left\{\mathcal{F}_{t}\right\}_{t \geq 0}, P\right)$ denote a complete probability space with a filtration $\left\{\mathcal{F}_{t}\right\}$ satisfying the usual conditions (i.e., it is increasing and right continuous while $\mathcal{F}_{0}$ contains all P-null sets), $B_{i}(t)$ are defined on this probability space.

We also introduce the following notations:

$$
\begin{aligned}
& \mathbb{R}_{+}^{d}=\left\{x \in \mathbb{R}_{+}: x_{i}>0, i=1,2, \ldots, d\right\} . \\
& \langle f\rangle_{t}=\frac{1}{t} \int_{0}^{t} f(s) \mathrm{ds}, \quad\langle f\rangle_{*}=\lim _{t \rightarrow \infty} \inf \frac{1}{t} \int_{0}^{t} f(s) \mathrm{ds}, \quad\langle f\rangle^{*}=\lim _{t \rightarrow \infty} \sup \frac{1}{t} \int_{0}^{t} f(s) \mathrm{ds} .
\end{aligned}
$$

Assume that $X(t) \in R^{+}$is an Itô-Lévy process of the form

$$
\mathrm{d} X(t)=F\left(X\left(t^{-}\right), t^{-}\right) \mathrm{d} t+G\left(X\left(t^{-}\right), t^{-}\right) \mathrm{d} B(t)+\int_{\mathbb{Y}} H\left(X\left(t^{-}\right), t^{-}, u\right) \tilde{N}(\mathrm{~d} t, \mathrm{~d} u)
$$

where $F: R^{n} \times R^{+} \times S \rightarrow R^{n}, G: R^{n} \times R_{+} \times S \rightarrow R^{n}$, and $H: R^{n} \times R_{+} \times S \times Y \rightarrow R^{n}$ are measurable functions.

Given $V \in C^{2,1}\left(R^{n} \times R^{+} \times S ; R^{+}\right)$, the operator $L V$ is defined by

$$
\begin{aligned}
L V(X, t)= & V_{t}(X, t)+V_{X}(X, t) F(X, t)+\frac{1}{2} \operatorname{trace}\left[G^{T}(X, t) V_{X X}(X, t) G(X, t)\right] \\
& +\int_{\mathbb{Y}}\left\{V(X+H(X, t))-V(X, t)-V_{X}(X, t) H(X, t, u)\right\} v(\mathrm{~d} u),
\end{aligned}
$$


where

$$
\begin{aligned}
& V_{t}(X, t)=\frac{\partial V_{X}(X, t)}{\partial t}, \quad V_{X}(X, t)=\left(\frac{\partial V_{X}(X, t)}{\partial X_{1}}, \ldots, \frac{\partial V_{X}(X, t)}{\partial X_{n}}\right), \\
& V_{X X}(X, t)=\left(\frac{\partial^{2} V_{X}(X, t)}{\partial X_{i} \partial X_{j}}\right)_{n \times n} .
\end{aligned}
$$

Then the generalized Itô's formula with Lévy jumps is given by

$$
\mathrm{d} V(X, t)=L V(X, t) \mathrm{d} t+V_{X}(X, t) G(X, t) \mathrm{d} B(t)+\int_{\mathbb{Y}}\{V(X+H(X, t))-V(X, t)\} \tilde{N}(\mathrm{~d} t, \mathrm{~d} u) .
$$

For convenience, we introduce the following lemmas and assumption which will be used later.

Assumption 1 We assume that $1+\gamma_{i}(u)>0, u \in Y(i=1,2,3)$, and there is a positive constant $c$ such that

$$
\int_{Y}\left[\ln \left(1+\gamma_{i}(u)\right)\right]^{2} v(\mathrm{~d} u)<c .
$$

Lemma 2.1 We assume that, for some $p>2, \mu^{*}-\frac{p-1}{2} \sigma^{2}-\frac{1}{p} \lambda^{*}>0$ holds. For any initial value $\left(x_{1}(0), x_{2}(0), x_{3}(0)\right) \in \mathbb{R}_{+}^{3}$, model (1.3) has a unique positive solution $\left(x_{1}(t), x_{2}(t)\right.$, $\left.x_{3}(t)\right) \in \mathbb{R}_{+}^{3}$ for any $t \geq 0$ almost surely. Furthermore, the solution $\left(x_{1}(t), x_{2}(t), x_{3}(t)\right)$ of model (1.3) has the following properties:

$$
\lim _{t \rightarrow \infty} \frac{\left(x_{1}(t)+2 x_{2}(t)+x_{3}(t)\right)}{t}=0 .
$$

Moreover,

$$
\lim _{t \rightarrow \infty} \frac{x_{1}(t)}{t}=0, \quad \lim _{t \rightarrow \infty} \frac{x_{2}(t)}{t}=0, \quad \lim _{t \rightarrow \infty} \frac{x_{3}(t)}{t}=0,
$$

where $\sigma^{2}=\sigma_{1}^{2} \vee \sigma_{2}^{2} \vee \sigma_{3}^{2}, \mu^{*}=\min \{\delta, a, \mu\}-\left(1-\eta_{\mathrm{PI}}\right) N a$, and $\lambda^{*}=\int_{Y}\left[\left(1+\gamma_{1}(u) \vee \gamma_{2}(u) \vee\right.\right.$ $\left.\left.\gamma_{3}(u)\right)^{p}-1-\gamma_{1}(u) \wedge \gamma_{2}(u) \wedge \gamma_{3}(u)\right] v(\mathrm{~d} u)$.

Proof Define $X=x_{1}+2 x_{2}+x_{3}, V=X^{p}$, applying Itô's formula, we get

$$
\begin{aligned}
\mathrm{d} V(X)= & L V(X) \mathrm{d} t+p X^{p-1}\left[\sigma_{1} \mathrm{~d} B_{1}(t)+\sigma_{2} \mathrm{~d} B_{2}(t)+\sigma_{3} \mathrm{~d} B_{3}(t)\right] \\
& +\int_{Y}\left[\left(x_{1}+\gamma_{1} x_{1}+2 x_{2}+2 \gamma_{2} x_{2}+x_{3}+\gamma_{3} x_{3}\right)^{p}-X^{p}\right] \tilde{N}(\mathrm{~d} t, \mathrm{~d} u),
\end{aligned}
$$

where

$$
\begin{aligned}
L V(X)= & p X^{p-1}\left[\lambda-\delta x_{1}-2 a x_{2}-\mu x_{3}+\left(1-\eta_{\mathrm{PI}}\right) \mathrm{Nax}_{2}\right] \\
& +\frac{p(p-1)}{2} X^{p-2}\left[\sigma_{1}^{2} x_{1}^{2}+4 \sigma_{2}^{2} x_{2}^{2}+\sigma_{3}^{2} x_{3}^{2}\right] \\
& +\int_{Y}\left[\left(x_{1}+\gamma_{1} x_{1}+2 x_{2}+2 \gamma_{2} x_{2}+x_{3}+\gamma_{3} x_{3}\right)^{p}-X^{p}\right.
\end{aligned}
$$




$$
\begin{aligned}
& \left.-p X^{p-1}\left(\gamma_{1} x_{1}+2 \gamma_{2} x_{2}+\gamma_{3} x_{3}\right)\right] v(\mathrm{~d} u) \\
\leq & p X^{p-1}\left[\lambda-\delta x_{1}-2 a x_{2}-\mu x_{3}+\left(1-\eta_{\mathrm{PI}}\right) N a x_{2}\right] \\
& +\frac{p(p-1)}{2} X^{p}\left(\sigma_{1}^{2}+\sigma_{2}^{2}+\sigma_{3}^{2}\right) \\
& +\int_{Y} X^{p}\left[\left(1+\gamma_{1} \vee \gamma_{2} \vee \gamma_{3}\right)^{p}-1-\left(\gamma_{1} \wedge \gamma_{2} \wedge \gamma_{3}\right)\right] v(\mathrm{~d} u) \\
\leq & p X^{p-2}\left[\lambda X-\left(\mu^{*}-\left(1-\eta_{\mathrm{PI}}\right) N a-\frac{p-1}{2} \sigma^{2}-\frac{1}{p} \lambda^{*}\right) X^{2}\right],
\end{aligned}
$$

where $\mu^{*}=\min \{\delta, a, \mu\}, p>2, b=\mu^{*}-\left(1-\eta_{\mathrm{PI}}\right) N a-\frac{p-1}{2} \sigma^{2}-\frac{1}{p} \lambda^{*}>0, \sigma^{2}=\sigma_{1}^{2} \vee \sigma_{2}^{2} \vee \sigma_{3}^{2}$, $\lambda^{*}=\int_{Y}\left[\left(1+\gamma_{1} \vee \gamma_{2} \vee \gamma_{3}\right)^{p}-1-\left(\gamma_{1} \wedge \gamma_{2} \wedge \gamma_{3}\right)\right] v(\mathrm{~d} u)$.

Thus, we have

$$
\begin{aligned}
\mathrm{d} V(X) \leq & p X^{p-2}\left(\lambda X-b X^{2}\right) \mathrm{d} t+p X^{p-1}\left[\sigma_{1} \mathrm{~d} B_{1}(t)+\sigma_{2} \mathrm{~d} B_{2}(t)\right. \\
& \left.+\sigma_{3} \mathrm{~d} B_{3}(t)\right]+\int_{Y} X^{p}\left[\left(1+\gamma_{1} \vee \gamma_{2} \vee \gamma_{3}\right)^{p}-1\right] \tilde{N}(\mathrm{~d} t, \mathrm{~d} u) .
\end{aligned}
$$

The following proof is similar to [29].

Lemma 2.2 We assume that, for some $p>2, \mu^{*}-\frac{p-1}{2} \sigma^{2}-\frac{1}{p} \lambda^{*}>0$ holds. For any initial value $\left(x_{1}(0), x_{2}(0), x_{3}(0)\right) \in \mathbb{R}_{+}^{3}$, model (1.3) has a unique positive solution $\left(x_{1}(t), x_{2}(t)\right.$, $\left.x_{3}(t)\right) \in \mathbb{R}_{+}^{3}$ for any $t \geq 0$ almost surely. Furthermore, the solution $\left(x_{1}(t), x_{2}(t), x_{3}(t)\right)$ of model (1.3) has the following properties:

$$
\begin{aligned}
& \lim _{t \rightarrow \infty} \sup \frac{\ln x_{1}(t)}{t} \leq 0, \quad \lim _{t \rightarrow \infty} \sup \frac{\ln x_{2}(t)}{t} \leq 0, \quad \lim _{t \rightarrow \infty} \sup \frac{\ln x_{3}(t)}{t} \leq 0, \quad \text { a.s. } \\
& \lim _{t \rightarrow \infty} \frac{\int_{0}^{t} x_{1}(s) \mathrm{d} B_{1}(s)}{t}=0, \quad \lim _{t \rightarrow \infty} \frac{\int_{0}^{t} x_{2}(s) \mathrm{d} B_{2}(s)}{t}=0, \\
& \lim _{t \rightarrow \infty} \frac{\int_{0}^{t} x_{3}(s) \mathrm{d} B_{3}(s)}{t}=0, \quad \text { a.s. } \\
& \lim _{t \rightarrow \infty} \frac{\int_{0}^{t} \int_{Y} \gamma_{1}(u) x_{1}(s) \tilde{N}(\mathrm{~d} s, \mathrm{~d} u)}{t}=0, \quad \lim _{t \rightarrow \infty} \frac{\int_{0}^{t} \int_{Y} \gamma_{2}(u) x_{2}(s) \tilde{N}(\mathrm{~d} s, \mathrm{~d} u)}{t}=0, \\
& \lim _{t \rightarrow \infty} \frac{\int_{0}^{t} \int_{Y} \gamma_{3}(u) x_{3}(s) \tilde{N}(\mathrm{~d} s, \mathrm{~d} u)}{t}=0, \quad \text { a.s., }
\end{aligned}
$$

where $\sigma^{2}=\sigma_{1}^{2} \vee \sigma_{2}^{2} \vee \sigma_{3}^{2}, \mu^{*}=\min \{\delta, a, \mu\}-\left(1-\eta_{\mathrm{PI}}\right) N a$, and $\lambda^{*}=\int_{Y}\left[\left(1+\gamma_{1}(u) \vee \gamma_{2}(u) \vee\right.\right.$ $\left.\left.\gamma_{3}(u)\right)^{p}-1-\gamma_{1}(u) \wedge \gamma_{2}(u) \wedge \gamma_{3}(u)\right] v(\mathrm{~d} u)$.

The proof of Lemma 2.2 is obtained based on ref [30], the Burkholder-Davis-Gundy inequality, and Hölder inequality.

Lemma 2.3 ([31]) Suppose that $Z(t) \in C\left(\Omega \times[0, \infty), \mathbb{R}_{+}\right)$. Under Assumption 1 ,

(I) if there are two positive constants $T$ and $\delta_{0}$ such that

$$
\ln Z(t) \leq \delta t-\delta_{0} \int_{0}^{t} Z(s) \mathrm{d} s+\sum_{i=1}^{n} \alpha_{i} B(t)+\sum_{i=1}^{n} k_{i} \int_{0}^{t} \int_{Y} \ln \left(1+\gamma_{i}(u)\right) \tilde{N}(\mathrm{~d} t, \mathrm{~d} u), \quad \text { a.s. }
$$


for all $t>T$, where $\alpha_{i}, \delta$, and $k_{i}$ are constants, then

$$
\left\{\begin{array}{lll}
\langle Z\rangle^{*} \leq \frac{\delta}{\delta_{0}} \quad \text { a.s., } & & \text { if } \delta \geq 0 \\
\lim _{t \rightarrow \infty} Z(t)=0 & \text { a.s., } & \text { if } \delta<0
\end{array}\right.
$$

(II) if there exist three positive constants $T, \delta$, and $\delta_{0}$ such that

$$
\ln Z(t) \geq \delta t-\delta_{0} \int_{0}^{t} Z(s) \mathrm{d} s+\sum_{i=1}^{n} \alpha_{i} B(t)+\sum_{i=1}^{n} k_{i} \int_{0}^{t} \int_{Y} \ln \left(1+\gamma_{i}(u)\right) \tilde{N}(\mathrm{~d} t, \mathrm{~d} u), \quad \text { a.s. }
$$

for all $t>T$, then $\langle Z\rangle_{*} \geq \frac{\delta}{\delta_{0}}$.

\section{Existence and global positive solution}

Theorem 3.1 For any given initial value $\left(x_{1}(0), x_{2}(0), x_{3}(0)\right) \in \mathbb{R}_{+}^{3}$, there is a unique positive solution $\left(x_{1}(t), x_{2}(t), x_{3}(t)\right)$ of model (1.3) on $t \geq 0$ and the solution will remain in $\mathbb{R}_{+}^{3}$ with probability 1 , namely $\left(x_{1}(t), x_{2}(t), x_{3}(t)\right) \in \mathbb{R}_{+}^{3}$ for all $t \geq 0$ almost surely.

Proof Since the coefficients of the equations are locally Lipschitz continuous, for any given initial value $\left(x_{1}(0), x_{2}(0), x_{3}(0)\right) \in \mathbb{R}_{+}^{3}$, there is a unique local solution $\left(x_{1}(t), x_{2}(t), x_{3}(t)\right)$ on $t \in\left[0, \tau_{e}\right)$, where $\tau_{e}$ is the explosion time. To show this solution is global, we need to show that $\tau_{e}=\infty$ a.s. At first, we prove $x_{1}(t), x_{2}(t)$, and $x_{3}(t)$ do not explode to infinity in a finite time. Set $m_{0}>0$ to be sufficiently large so that all $x_{1}(0), x_{2}(0)$, and $x_{3}(0)$ lie within the interval $\left[\frac{1}{m_{0}}, m_{0}\right]$. For each integer $m \geq m_{0}$, define the stopping time

$$
\tau_{m}=\inf \left\{t \in\left[0, \tau_{e}\right): x_{1}(t) \notin\left(\frac{1}{m}, m\right) \text { or } x_{2}(t) \notin\left(\frac{1}{m}, m\right) x_{3}(t) \notin\left(\frac{1}{m}, m\right)\right\} .
$$

Clearly, $\tau_{m}$ is increasing as $m \rightarrow \infty$ a.s. Set $\tau_{\infty}=\lim _{m \rightarrow \infty} \tau_{m}$, where $\tau_{\infty} \leq \tau_{e}$ a.s. If we can show that $\tau_{\infty}=\infty$ is true, then $\tau_{e}=\infty$ and $\left(x_{1}(t), x_{2}(t), x_{3}(t)\right) \in \mathbb{R}_{+}^{3}$ a.s. If this statement is false, then there exist a pair of constants $T>0$ and $0<\varepsilon<1$ such that

$$
P\left(\tau_{\infty} \leq T\right) \geq \varepsilon
$$

Hence, there is an integer $m_{1} \geq m_{0}$ such that

$$
P\left(\tau_{m} \leq T\right) \geq \varepsilon \quad \text { for all } m_{1} \geq m_{0} .
$$

Define a $C^{3}$-function by

$$
V\left(x_{1}, x_{2}, x_{3}\right)=\left(x_{1}+a_{1}-\ln \frac{x_{1}}{a_{1}}\right)+\left(x_{2}+1-\ln x_{2}\right)+a_{2}\left(x_{3}+1-\ln x_{3}\right),
$$

where $a_{1}, a_{2}$ are positive constants to be defined later. The nonnegativity of this function can be seen from

$$
(u-1-\ln u) \geq 0 \quad \text { for } u \geq 0 .
$$


Let $m \geq m_{0}$ and $T>0$ be arbitrary. Using Itô's formula [32], we get

$$
\begin{aligned}
\mathrm{d} V\left(x_{1}, x_{2}, x_{3}\right)= & L V\left(x_{1}, x_{2}\right) \mathrm{d} t+\sigma_{1}\left(x_{1}-a_{1}\right) \mathrm{d} B_{1}(t)+\sigma_{2}\left(x_{2}-1\right) \mathrm{d} B_{2}(t) \\
& +a_{2} \sigma_{3}\left(x_{3}-1\right) \mathrm{d} B_{3}(t)+\int_{Y}\left[\gamma_{1}(u) x_{1}-a_{1} \ln \left(1+\gamma_{1}(u)\right)\right. \\
& \left.+\gamma_{2}(u) x_{2}-\ln \left(1+\gamma_{2}(u)\right)+a_{2} \gamma_{3}(u) x_{3}-a_{2} \ln \left(1+\gamma_{3}(u)\right)\right] \tilde{N}(\mathrm{~d} t, \mathrm{~d} u),
\end{aligned}
$$

where

$$
\begin{aligned}
L V\left(x_{1}, x_{2}, x_{3}\right)= & \left(\left(1-\gamma_{\mathrm{RTI}}\right) \beta a_{2}-\delta\right) x_{1}+\left(\left(1-\eta_{\mathrm{PI}}\right) N a a_{2}-a\right) x_{2} \\
& +\left(\left(1-\gamma_{\mathrm{RTI}}\right) \beta a_{1}-a_{2} \mu\right) x_{3}+\lambda+a_{1} \delta+a+a_{2} \mu \\
& +\frac{1}{2} a_{1} \sigma_{1}^{2}+\frac{1}{2} \sigma_{2}^{2}+\frac{1}{2} a_{2} \sigma_{3}^{2}-\left(1-\gamma_{\mathrm{RTI}}\right) \beta a_{2} x_{1} x_{3} \\
& -\frac{a_{1} \lambda}{x_{1}}-\frac{\left(1-\gamma_{\mathrm{RTI}}\right) \beta x_{1} x_{3}}{x_{2}}-\frac{\left(1-\eta_{\mathrm{PI}}\right) a_{2} N a x_{2}}{x_{3}} \\
& +\int_{Y}\left[a_{1} \gamma_{1}(u)-a_{1} \ln \left(1+\gamma_{1}(u)\right)+\gamma_{2}(u)\right. \\
& \left.-\ln \left(1+\gamma_{2}(u)\right)+a_{2} \gamma_{3}(u)-a_{2} \ln \left(1+\gamma_{3}(u)\right)\right] v(\mathrm{~d} u) \\
\leq & \left(1-\gamma_{\mathrm{RTI}}\right) \beta\left(a_{2}-\frac{\delta}{\left(1-\gamma_{\mathrm{RTI}}\right) \beta}\right) x_{1}+\left(1-\eta_{\mathrm{PI}}\right) N a\left(a_{2}-\frac{1}{\left(1-\eta_{\mathrm{PI}}\right) N}\right) x_{2} \\
& +\left(1-\gamma_{\mathrm{RTI}}\right) \beta\left(a_{1}-\frac{a_{2} u}{\left(1-\gamma_{\mathrm{RTI}}\right) \beta}\right) x_{3} \\
& +\lambda+a_{1} \delta+a+a_{2} \mu+\frac{1}{2} a_{1} \sigma_{1}^{2}+\frac{1}{2} \sigma_{2}^{2}+\frac{1}{2} a_{2} \sigma_{3}^{2}+3 K_{1} \\
\leq & \lambda+a_{1} \delta+a+a_{2} \mu+\frac{1}{2} a_{1} \sigma_{1}^{2}+\frac{1}{2} \sigma_{2}^{2}+\frac{1}{2} a_{2} \sigma_{3}^{2}+3 K_{1} \\
=: & M,
\end{aligned}
$$

where $a_{1}=\frac{a_{2} u}{\left(1-\gamma_{\mathrm{RTI}}\right) \beta}, a_{2}=\min \left\{\frac{\delta}{\left(1-\gamma_{\mathrm{RTI}}\right) \beta}, \frac{1}{\left(1-\eta_{\mathrm{PI}}\right) N}\right\}$, so we have $a_{1}-\frac{a_{2} u}{\left(1-\gamma_{\mathrm{RTI}}\right) \beta}=0, a_{2}-$ $\frac{\delta}{\left(1-\gamma_{\mathrm{RTI}}\right) \beta} \leq 0$, and $a_{2}-\frac{1}{\left(1-\eta_{\mathrm{PI}}\right) N} \leq 0$, and $K_{1}=\max \left\{a_{1} \int_{Y}\left[\gamma_{1}(u)-\ln \left(1+\gamma_{1}(u)\right)\right] v(\mathrm{~d} u)\right.$, $\left.\int_{Y}\left[\gamma_{2}(u)-\ln \left(1+\gamma_{2}(u)\right)\right] v(\mathrm{~d} u), a_{2} \int_{Y}\left[\gamma_{3}(u)-\ln \left(1+\gamma_{3}(u)\right)\right] v(\mathrm{~d} u)\right\}$.

The proof of the remaining part follows the proof in Ref. [26].

\section{Extinction of the disease}

For HIV infection, the main concern is the conditions which make the infected CD4 ${ }^{+}$ $\mathrm{T}$-cells and free virus particles eradicate in a long term. In this section, we will give a result of extinction of infected $\mathrm{CD} 4^{+} \mathrm{T}$-cells and free virus particles in stochastic model with Lévy noise.

Denote

$$
\tilde{R}_{0}=\frac{\left[\left(\left(1-\eta_{\mathrm{PI}}\right) N a-a\right)-\mu\right]^{2}}{\left(\sigma_{2}^{2}+2 \mu\right)\left(\sigma_{1}^{2}-2\left[\left(1-\eta_{\mathrm{PI}}\right) N a-a\right]\right)} .
$$


Theorem 4.1 Assume that $\sigma_{1}^{2}-2\left[\left(1-\eta_{\mathrm{PI}}\right) N a-a\right]>0$, then for any given initial value $\left(x_{1}(0), x_{2}(0), x_{3}(0)\right) \in \mathbb{R}_{+}^{3}$, the solution $\left(x_{1}(t), x_{2}(t), x_{3}(t)\right) \in \mathbb{R}_{+}^{3}$ of model $(1.3)$ has the property

$$
\lim _{t \rightarrow \infty} \sup \frac{\ln \left(x_{2}+x_{3}\right)}{t} \leq-\frac{1}{4}\left|\lambda_{\max }\right|<0
$$

if $\tilde{R}_{0}<1$ holds.

Proof Applying Itô's formula, we can conclude that

$$
\begin{aligned}
& \mathrm{d} \ln \left(x_{2}+x_{3}\right) \\
& =\frac{1}{2\left(x_{2}+x_{3}\right)^{2}}\left\{2\left(x_{2}+x_{3}\right)\left[\left(1-\eta_{\mathrm{PI}}\right) \operatorname{Nax}_{2}-a x_{2}-\mu x_{3}\right]\right. \\
& \left.-\sigma^{2} x_{2}^{2}-\sigma^{3} x_{3}^{2}+\beta_{3}\right\} \mathrm{d} t+\frac{1}{x_{2}+x_{3}}\left[\sigma_{2} x_{2} \mathrm{~d} B_{2}(t)+\sigma_{3} x_{3} \mathrm{~d} B_{3}(t)\right] \\
& +\int_{Y} \ln \left(1+\frac{x_{2} \gamma_{2}(u)+x_{3} \gamma_{3}(u)}{x_{2}+x_{3}}\right) \tilde{\mathrm{N}}(\mathrm{d} t, \mathrm{~d} u) \\
& =\frac{1}{2\left(x_{2}+x_{3}\right)^{2}}\left\{\left[\begin{array}{ll}
x_{2} & x_{3}
\end{array}\right]\right. \\
& \left.\times\left[\begin{array}{cc}
2 a\left(\left(1-\eta_{\mathrm{PI}}\right) N-1\right)-\sigma_{1}^{2} & a\left(N\left(1-\eta_{\mathrm{PI}}\right)-1\right)-\mu \\
a\left(N\left(1-\eta_{\mathrm{PI}}\right)-1\right)-\mu & -2 \mu-\sigma_{2}^{2}
\end{array}\right]\left[\begin{array}{l}
x_{2} \\
x_{3}
\end{array}\right]\right\} \mathrm{d} t \\
& +\frac{\beta_{3}}{2\left(x_{2}+x_{3}\right)^{2}} \mathrm{~d} t+\frac{1}{x_{2}+x_{3}}\left[\sigma_{2} x_{2} \mathrm{~d} B_{2}(t)+\sigma_{3} x_{3} \mathrm{~d} B_{3}(t)\right] \\
& +\int_{Y} \ln \left(1+\frac{x_{2} \gamma_{2}(u)+x_{3} \gamma_{3}(u)}{x_{2}+x_{3}}\right) \tilde{\mathrm{N}}(\mathrm{d} t, \mathrm{~d} u)
\end{aligned}
$$

where $\beta_{3}=\int_{Y} \ln \left[\left(1+\frac{x_{2} \gamma_{2}(u)+x_{3} \gamma_{3}(u)}{x_{2}+x_{3}}\right)-\frac{x_{2} \gamma_{2}(u)+x_{3} \gamma_{3}(u)}{x_{2}+x_{3}}\right] v(\mathrm{~d} u)<0$.

Let

$$
A=\left[\begin{array}{cc}
2 a\left(\left(1-\eta_{\mathrm{PI}}\right) N-1\right)-\sigma_{1}^{2} & a\left(N\left(1-\eta_{\mathrm{PI}}\right)-1\right)-\mu \\
a\left(N\left(1-\eta_{\mathrm{PI}}\right)-1\right)-\mu & -2 \mu-\sigma_{2}^{2}
\end{array}\right],
$$

if conditions

$$
\sigma_{1}^{2}-2 a\left(\left(1-\eta_{\mathrm{PI}}\right) N-1\right)>0
$$

and

$$
|A|=-\left(2 \mu+\sigma_{2}^{2}\right)\left(\sigma_{1}^{2}-2\left[\left(1-\eta_{\mathrm{PI}}\right) N a-a\right]\right)\left(\tilde{R}_{0}-1\right)>0
$$

are satisfied, then we can conclude that the matrix $A$ is negative-definite, with the largest (negative) eigenvalue $\lambda_{\max }$. Then we have

$$
\begin{aligned}
\mathrm{d} \ln \left(x_{2}+x_{3}\right) \leq & -\frac{1}{4}\left|\lambda_{\max }\right| \mathrm{d} t+\frac{1}{x_{2}+x_{3}}\left[\sigma_{2} x_{2} \mathrm{~d} B_{2}(t)+\sigma_{3} x_{3} \mathrm{~d} B_{3}(t)\right] \\
& +\int_{Y} \ln \left(1+\frac{x_{2} \gamma_{2}(u)+x_{3} \gamma_{3}(u)}{x_{2}+x_{3}}\right) \tilde{\mathrm{N}}(\mathrm{d} t, \mathrm{~d} u) .
\end{aligned}
$$


Integrating from 0 to $t$ on both sides of (4.1) yields

$$
\begin{aligned}
\frac{\ln \left(x_{2}(t)+x_{3}(t)\right)}{t} \leq & -\frac{1}{4}\left|\lambda_{\max }\right|+\frac{\sigma_{2}}{t} \int_{0}^{t} \frac{x_{2}}{x_{2}+x_{3}} \mathrm{~d} B_{2}(s)+\frac{\sigma_{3}}{t} \int_{0}^{t} \frac{x_{3}}{x_{2}+x_{3}} \mathrm{~d} B_{3}(s) \\
& +\frac{1}{t} \int_{0}^{t} \int_{Y} \ln (1+\gamma(u)) \tilde{\mathrm{N}}(\mathrm{d} s, \mathrm{~d} u)+\frac{\ln \left(x_{2}(0)+x_{3}(0)\right)}{t},
\end{aligned}
$$

where $\gamma(u)=\gamma_{2}(u) \vee \gamma_{3}(u)$.

Combined with Lemma 2.1, Lemma 2.2, and Lemma 2.3, clearly,

$$
\limsup _{t \rightarrow \infty} \frac{\ln \left(x_{2}+x_{3}\right)}{t} \leq-\frac{1}{4}\left|\lambda_{\max }\right|<0
$$

The proof is completed.

Remark 4.1 The sufficient criteria of extinction are established for the infected CD4 ${ }^{+}$ T-cells and free virus particles in the stochastic model with Lévy noise. From Theorem 4.1, we can obtain that strong fluctuation in internal HIV viral dynamics accelerates the extinction of the infected $\mathrm{CD} 4^{+} \mathrm{T}$-cells and free virus particles.

\section{Persistence in mean}

For simplicity, we introduce the following notation:

$$
\left\langle x_{i}(t)\right\rangle=\frac{1}{t} \int_{0}^{t} x_{i}(s) \mathrm{d} s, \quad i=1,2,3
$$

Definition 5.1 Model (1.3) is said to be persistent in the mean if

$$
\begin{aligned}
& \lim _{t \rightarrow \infty} \inf \frac{1}{t} \int_{0}^{t} x_{2}(s) \mathrm{d} s>0, \\
& \lim _{t \rightarrow \infty} \inf \frac{1}{t} \int_{0}^{t} x_{3}(s) \mathrm{d} s>0 \quad \text { a.s. }
\end{aligned}
$$

Denote

$$
\tilde{R}_{1}=\frac{\lambda\left(1-\gamma_{\mathrm{RTI}}\right) \beta\left(1-\eta_{\mathrm{PI}}\right) N a}{\left(\delta+\bar{\sigma}_{1}\right)\left(a+\bar{\sigma}_{2}\right)\left(\mu+\frac{\left(1-\gamma_{\mathrm{RTI}}\right) \beta}{\delta}+\bar{\sigma}_{3}\right)} .
$$

Theorem 5.1 Assume that $\left(1-\eta_{\mathrm{PI}}\right) N-1>0$, then for any solution $\left(x_{1}(t), x_{2}(t), x_{3}(t)\right) \in \mathbb{R}_{+}^{3}$ of model (1.3) with initial value $\left(x_{1}(0), x_{2}(0), x_{3}(0)\right) \in \mathbb{R}_{+}^{3}$, if $\tilde{R}_{1}>1$, then

$$
\begin{aligned}
& \lim _{t \rightarrow \infty} \inf \left|x_{3}(t)\right\rangle \geq \frac{\rho}{\beta\left(1-\gamma_{\mathrm{RTI}}\right)}, \\
& \lim _{t \rightarrow \infty} \inf \left|x_{2}(t)\right\rangle \geq \frac{\mu \rho}{a \beta\left(1-\gamma_{\mathrm{RTI}}\right)\left[\left(1-\eta_{\mathrm{PI}}\right) N-1\right]} .
\end{aligned}
$$


Proof Define $V\left(x_{1}, x_{2}, x_{3}\right)=-\ln x_{1}-c_{1} \ln x_{2}-c_{2}\left(\ln x_{3}-\frac{\left(1-\gamma_{\mathrm{RTI}}\right) \beta}{\delta} x_{1}\right)$. Applying Itô's formula, we obtain

$$
\begin{aligned}
\mathrm{d} V\left(x_{1}, x_{2}, x_{3}\right)= & L V \mathrm{~d} t-\sigma_{1}\left(1-\frac{c_{2}\left(1-\gamma_{\mathrm{RTI}}\right) \beta}{\delta}\right) \mathrm{d} B_{1}(t)-c_{1} \sigma_{2} \mathrm{~d} B_{2}(t) \\
& -c_{2} \sigma_{3} \mathrm{~d} B_{3}(t)-\int_{Y}\left[\ln \left(1+\gamma_{1}(u)\right)+c_{1} \ln \left(1+\gamma_{2}(u)\right)\right. \\
& \left.+c_{2} \ln \left(1+\gamma_{3}(u)\right)-\frac{c_{2}\left(1-\gamma_{\mathrm{RTI}}\right) \beta}{\delta} \ln \gamma_{1}(u) x_{1}(t)\right] \tilde{N}(\mathrm{~d} t, \mathrm{~d} u),
\end{aligned}
$$

where

$$
\begin{aligned}
L V= & {\left[-\frac{\lambda}{x_{1}}-c_{1}\left(1-\gamma_{\mathrm{RTI}}\right) \beta \frac{x_{1} x_{3}}{x_{2}}-\frac{c_{2}\left(1-\eta_{\mathrm{PI}}\right) N a x_{2}}{x_{3}}+\delta\right.} \\
& +\left(1-\gamma_{\mathrm{RTI}}\right) \beta x_{3}+\frac{1}{2} \sigma_{1}^{2}+a c_{1}+\frac{c_{1}}{2} \sigma_{2}^{2}+c_{2} \mu+\frac{c_{2}}{2} \sigma_{3}^{2} \\
& \left.+\frac{c_{2} \lambda\left(1-\gamma_{\mathrm{RTI}}\right) \beta}{\delta}-\frac{c_{2}\left(1-\gamma_{\mathrm{RTI}}\right)^{2} \beta^{2} x_{1} x_{3}}{\delta}\right] \\
& +\int_{Y}\left[\gamma_{1}(u)-\ln \left(1+\gamma_{1}(u)\right)+c_{1}\left(\gamma_{2}(u)-\ln \left(1+\gamma_{2}(u)\right)\right)\right. \\
& \left.+c_{2}\left(\gamma_{3}(u)-\ln \left(1+\gamma_{3}(u)\right)\right)\right] v(\mathrm{~d} u) \\
\leq & -3 \sqrt[3]{\lambda c_{1}\left(1-\gamma_{\mathrm{RTI}}\right) \beta\left(1-\eta_{\mathrm{PI}}\right) N a c_{2}} \\
& +c_{1}\left(a+\frac{\sigma_{2}^{2}}{2}+\int \gamma_{Y}\left[\gamma_{2}(u)-\ln \left(1+\gamma_{2}(u)\right)\right] v(\mathrm{~d} u)\right) \\
& +c_{2}\left(\mu+\frac{\sigma_{3}^{2}}{2}+\frac{\left(1-\gamma_{\mathrm{RTI}}\right) \beta}{\delta}+\int_{Y}\left[\gamma_{3}(u)-\ln \left(1+\gamma_{3}(u)\right)\right] v(\mathrm{~d} u)\right) \\
& +\delta+\left(1-\gamma_{\mathrm{RTI}}\right) \beta x_{3}+\frac{1}{2} \sigma_{1}^{2}+\int_{Y}\left[\gamma_{1}(u)-\ln \left(1+\gamma_{1}(u)\right)\right] v(\mathrm{~d} u) .
\end{aligned}
$$

Note $\bar{\sigma}_{1}=\frac{1}{2} \sigma_{1}^{2}+\int_{Y}\left[\gamma_{1}(u)-\ln \left(1+\gamma_{1}(u)\right)\right] v(\mathrm{~d} u), \bar{\sigma}_{2}=\frac{1}{2} \sigma_{2}^{2}+\int_{Y}\left[\gamma_{2}(u)-\ln \left(1+\gamma_{2}(u)\right)\right] v(\mathrm{~d} u)$, and $\bar{\sigma}_{3}=\frac{1}{2} \sigma_{3}^{2}+\int_{Y}\left[\gamma_{3}(u)-\ln \left(1+\gamma_{3}(u)\right)\right] v(\mathrm{~d} u)$, then let

$$
c_{1}\left(a+\bar{\sigma}_{2}\right)=c_{2}\left(\mu+\frac{\left(1-\gamma_{\mathrm{RTI}}\right) \beta}{\delta}+\bar{\sigma}_{3}\right)=\frac{\lambda\left(1-\gamma_{\mathrm{RTI}}\right) \beta\left(1-\eta_{\mathrm{PI}}\right) N a}{\left(a+\bar{\sigma}_{2}\right)\left(\mu+\frac{\left(1-\gamma_{\mathrm{RTI}}\right) \beta}{\delta}+\bar{\sigma}_{2}\right)},
$$

we have

$$
c_{1}=\frac{\lambda\left(1-\gamma_{\mathrm{RTI}}\right) \beta\left(1-\eta_{\mathrm{PI}}\right) N a}{\left(a+\bar{\sigma}_{2}\right)^{2}\left(\mu+\frac{\left(1-\gamma_{\mathrm{RTI}}\right) \beta}{\delta}+\bar{\sigma}_{2}\right)}, \quad c_{2}=\frac{\lambda\left(1-\gamma_{\mathrm{RTI}}\right) \beta\left(1-\eta_{\mathrm{PI}}\right) N a}{\left(a+\bar{\sigma}_{2}\right)\left(\mu+\frac{\left(1-\gamma_{\mathrm{RTI}}\right) \beta}{\delta}+\bar{\sigma}_{2}\right)^{2}}
$$

So,

$$
\begin{aligned}
L V & \leq-\frac{\lambda\left(1-\gamma_{\mathrm{RTI}}\right) \beta\left(1-\eta_{\mathrm{PI}}\right) N a}{\left(a+\bar{\sigma}_{2}\right)\left(\mu+\frac{\left(1-\gamma_{\mathrm{RTI}}\right) \beta}{\delta}+\bar{\sigma}_{2}\right)}+\delta+\bar{\sigma}_{1}+\left(1-\gamma_{\mathrm{RTI}}\right) \beta x_{3} \\
& =-\rho+\left(1-\gamma_{\mathrm{RTI}}\right) \beta x_{3},
\end{aligned}
$$


where

$$
\begin{aligned}
\rho & =\left(\delta+\bar{\sigma}_{1}\right)\left[\frac{\lambda\left(1-\gamma_{\mathrm{RTI}}\right) \beta\left(1-\eta_{\mathrm{PI}}\right) N a}{\left(a+\bar{\sigma}_{2}\right)\left(\mu+\frac{\left(1-\gamma_{\mathrm{RTI}}\right) \beta}{\delta}+\bar{\sigma}_{2}\right)\left(\delta+\bar{\sigma}_{1}\right)}-1\right] \\
& =\left(\delta+\bar{\sigma}_{1}\right)\left(\tilde{R}_{1}-1\right) .
\end{aligned}
$$

We obtain that

$$
\begin{aligned}
\mathrm{d} V\left(x_{1}, x_{2}, x_{3}\right)< & -\rho \mathrm{d} t+\left(1-\gamma_{\mathrm{RTI}}\right) \beta x_{3} \mathrm{~d} t+\sigma_{1}\left(1-\frac{c_{2}\left(1-\gamma_{\mathrm{RTI}}\right) \beta}{\delta}\right) \mathrm{d} B_{1}(t) \\
& -c_{1} \sigma_{2} \mathrm{~d} B_{2}(t)-c_{2} \sigma_{3} \mathrm{~d} B_{3}(t)-\int_{Y}\left[\ln \left(1+\gamma_{1}(u)\right)+c_{1} \ln \left(1+\gamma_{2}(u)\right)\right. \\
& \left.+c_{2} \ln \left(1+\gamma_{3}(u)\right)-\frac{c_{2}\left(1-\gamma_{\mathrm{RTI}}\right) \beta}{\delta} \gamma_{1}(u) x_{1}(t)\right] \tilde{N}(\mathrm{~d} t, \mathrm{~d} u) .
\end{aligned}
$$

Let $c_{3}=\frac{c_{2}\left(1-\gamma_{\mathrm{RTI}}\right) \beta}{\delta}$, when $\rho \geq 0$, that is, $\tilde{R}_{1}>1$. Integrating from 0 to $t$ on both sides of (5.1), we obtain

$$
\begin{aligned}
& \frac{\ln V\left(x_{1}(t), x_{2}(t), x_{3}(t)\right)-\ln V\left(x_{1}(0), x_{2}(0), x_{3}(0)\right)}{t} \\
& \leq-\rho+\left(1-\gamma_{\mathrm{RTI}}\right) \beta\left\langle x_{3}(t)\right\rangle-\frac{\left(1-c_{3}\right) \sigma_{1}}{t} \int_{0}^{t} B_{1}(s) \mathrm{d} s-\frac{c_{1} \sigma_{2}}{t} \int_{0}^{t} B_{2}(s) \mathrm{d} s \\
& \quad-\frac{c_{2} \sigma_{3}}{t} \int_{0}^{t} B_{3}(s) \mathrm{d} s-\frac{1}{t} \int_{0}^{t} \int_{Y}\left[\ln \left(1+\gamma_{1}(u)\right)+c_{1} \ln \left(1+\gamma_{2}(u)\right)\right. \\
& \left.\quad+c_{2} \ln \left(1+\gamma_{3}(u)\right)+c_{3} \gamma_{1}(u) x_{1}(s)\right] \tilde{N}(\mathrm{~d} s, \mathrm{~d} u) .
\end{aligned}
$$

Take the limit on both sides of (5.2), considering Lemma 2.1, Lemma 2.2, and Lemma 2.3, we have

$$
\lim _{t \rightarrow \infty} \frac{\int_{0}^{t} B_{i}(s) \mathrm{d} s}{t}=0, \quad i=1,2,3,
$$

and

$$
\lim _{t \rightarrow \infty}\left\langle x_{3}(t)\right\rangle \geq \frac{\rho}{\beta\left(1-\gamma_{\mathrm{RTI}}\right)} .
$$

The same as

$$
\lim _{t \rightarrow \infty} \inf \left|x_{3}(t)\right\rangle \geq \frac{\rho}{\beta\left(1-\gamma_{\mathrm{RTI}}\right)} .
$$

On the other hand, applying Itô's formula, we can conclude that

$$
\mathrm{d}\left(x_{2}+x_{3}\right)=L V \mathrm{~d} t+\int_{Y}\left[\gamma_{2}(u) x_{2}(t)+\gamma_{3}(u) x_{3}(t)\right] \tilde{N}(\mathrm{~d} t, \mathrm{~d} u)
$$

where

$$
L V=\left[\left(\left(1-\eta_{\mathrm{PI}}\right) N a-a\right) x_{2}-\mu x_{3}\right] \mathrm{d} t+\sigma_{2} x_{2} \mathrm{~d} B_{2}(t)+\sigma_{3} x_{3} \mathrm{~d} B_{3}(t) .
$$


Integrating both sides of (5.5) from 0 to $t$ yields

$$
\begin{aligned}
\frac{x_{2}(t)}{t}+\frac{x_{3}(t)}{t}= & \left(\left(1-\eta_{\mathrm{PI}}\right) N a-a\right)\left\langle x_{2}(t)\right\rangle-\mu\left\langle x_{3}(t)\right\rangle \\
& +\frac{1}{t} \int_{0}^{t} \int_{Y}\left(\gamma_{2}(u) x_{2}(s)+\gamma_{3}(u) x_{3}(s)\right) \tilde{N}(\mathrm{~d} s, \mathrm{~d} u) \\
& +\frac{\sigma_{2} x_{2} B_{2}(t)}{t}+\frac{\sigma_{3} x_{3} B_{3}(t)}{t}+\frac{x_{2}(0)}{t}+\frac{x_{3}(0)}{t} .
\end{aligned}
$$

Clearly, we can derive that

$$
\left\langle x_{2}(t)\right\rangle=\frac{\mu}{\left(1-\eta_{\mathrm{PI}}\right) N a-a}\left\langle x_{3}(t)\right\rangle+\phi(t),
$$

where

$$
\begin{aligned}
\phi(t)= & -\left[\frac{x_{2}(t)-x_{2}(0)}{t}+\frac{x_{3}(t)-x_{3}(0)}{t}-\frac{\int_{0}^{t} \sigma_{2} x_{2}(s) \mathrm{d} B_{2}(s)}{t}\right. \\
& \left.-\int_{0}^{t} \frac{\sigma_{3} x_{3}(s) \mathrm{d} B_{3}(s)}{t}-\frac{1}{t} \int_{0}^{t} \int_{Y}\left(\gamma_{2}(u) x_{2}(s)+\gamma_{3}(u) x_{3}(s)\right) \tilde{N}(\mathrm{~d} s, \mathrm{~d} u)\right] .
\end{aligned}
$$

According to Lemma 2.1 and Lemma 2.2, we have

$$
\lim _{t \rightarrow \infty} \phi(t)=0 .
$$

Together with (5.4) and (5.6), we obtain

$$
\lim _{t \rightarrow \infty}\left\langle x_{2}(t)\right\rangle \geq \frac{\mu \rho}{a \beta\left(1-\gamma_{\mathrm{RTI}}\right)\left[\left(1-\eta_{\mathrm{PI}}\right) N-1\right]} .
$$

The same as

$$
\lim _{t \rightarrow \infty} \inf \left|x_{2}(t)\right\rangle \geq \frac{\mu \rho}{a \beta\left(1-\gamma_{\mathrm{RTI}}\right)\left[\left(1-\eta_{\mathrm{PI}}\right) N-1\right]} .
$$

Therefore, if $\tilde{R_{1}}>1$, we get the conclusion of Theorem 5.1. So, the proof is completed.

Remark 5.1 From the expression of $\tilde{R_{1}}$, we can realize that the persistence of the infected CD4+ cells and virus particles depend not only on the highly active antiretroviral treatment (HAART), but also on the stochastic fluctuation intensity of the biochemical circumstance. Obviously, $\tilde{R_{1}} \leq R_{0}$, if and only if $\overline{\sigma_{1}}=0, \overline{\sigma_{2}}=0, \overline{\sigma_{3}}=0$, then $\tilde{R}_{1}=R_{0}$. This means that strong stochastic fluctuation can suppress the replication of virus particles and the release of new particles.

\section{Numerical results}

In this section, the results of various simulations of HIV infection dynamics are presented. By an order $\frac{1}{2}$ Euler scheme approximation [33,34], we discuss the effect of white noises and Lévy noise on the viral dynamics.

Firstly, we verify the extinction of system (1.3), choose parameters as in Table 1, the intensity of noise $\left(\sigma_{1}, \sigma_{2}, \sigma_{3}\right)=(4.2,4,1.8),\left(\gamma_{1}, \gamma_{2}, \gamma_{3}\right)=(1.2,0.9,1.4) N=20$. For stochastic 
Table 1 List of parameters

\begin{tabular}{llll}
\hline Parameter & Description & Value & Source \\
\hline$\lambda$ & Production rate of new target cells & $2 \times 10^{5} /$ day & {$[35]$} \\
$\delta$ & Death rate of uninfected cells & $0.1 /$ uninfected cell/day & {$[35]$} \\
$\beta$ & Infection rate of uninfected cells & $2.4 \times 10^{-7} /$ virus/uninfected cell/day & {$[35]$} \\
$a$ & Death rate of infected cells and viral lysis & $0.5 /$ infected cell/day & {$[5,35]$} \\
$\mu$ & Virion clearance rate & $5 /$ virion/day & {$[35]$} \\
$\gamma_{\text {RTI }}$ & The efficacy of reverse transcriptase inhibitor & 0.5 & {$[12]$} \\
& drug effect & & {$[12]$} \\
$\eta_{\text {PI }}$ & The efficacy of protease inhibitor drug & 0.12 & \\
\hline
\end{tabular}

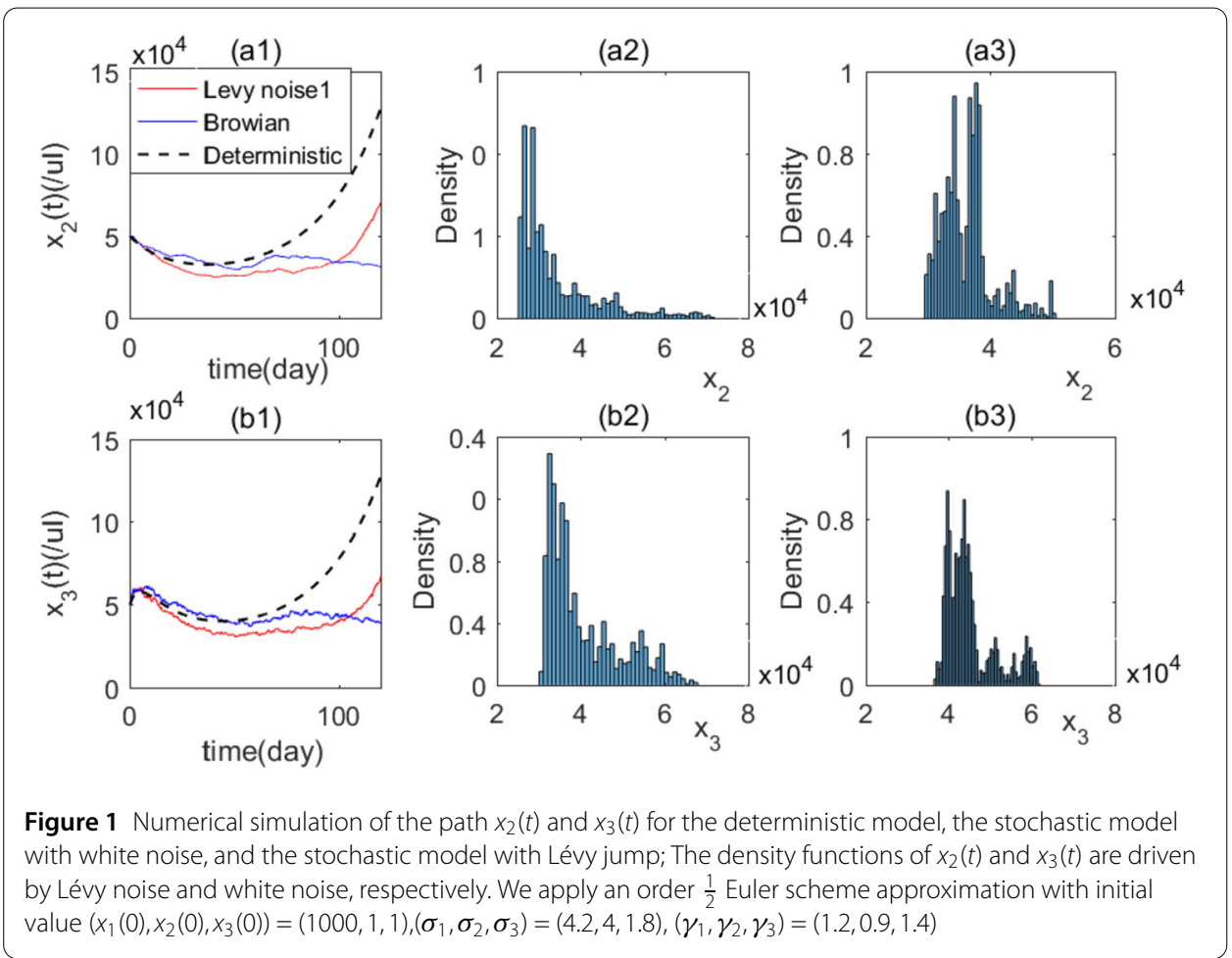

model with Lévy jump, we obtain $\tilde{R}_{0}=0.42<1$. Figure 1 (a1) and Fig. 1 (b1) show that the solutions of infected CD4+ cells and virus particles will tend to zero with probability 1 , respectively. The probability density of the values of $x_{2}$ and $x_{3}$ are shown in Fig. 1(a2) and Fig. 1(b2) driven by Lévy noise and Fig. 1(a3) and (b3) affected by white noise. By comparing the results, we found that strong fluctuation will result in faster extinction of infected CD4+ cells and virus particles than white noise. While we set $\left(\sigma_{1}, \sigma_{2}, \sigma_{3}\right)=(0.55,0.4,0.7)$, $\left(\gamma_{1}, \gamma_{2}, \gamma_{3}\right)=(0.2,0.3,0.4), N=80$, then $\tilde{R}_{1}>1$, from Fig. 2 we can see that the infected $\mathrm{CD} 4+$ cells and virus particles are both persistent in mean.

Next, we assume that the intensity of noise is $\left(\sigma_{1}, \sigma_{2}, \sigma_{3}\right)=(10,5,1.8)$ and $\left(\gamma_{1}, \gamma_{2}, \gamma_{3}\right)=$ $(1.6,1.3,1.4), N=80$, there are some interesting results found in the numerical simulations, see Fig. 3. For the stochastic model with Lévy jump, we obtain $\tilde{R}_{0}=0.84<1$, the infected CD4+ cells and virus particles are both extinct, while for the deterministic model $R_{0}=2.9>1$, the infected CD4+ cells and virus particles are both persistent. 

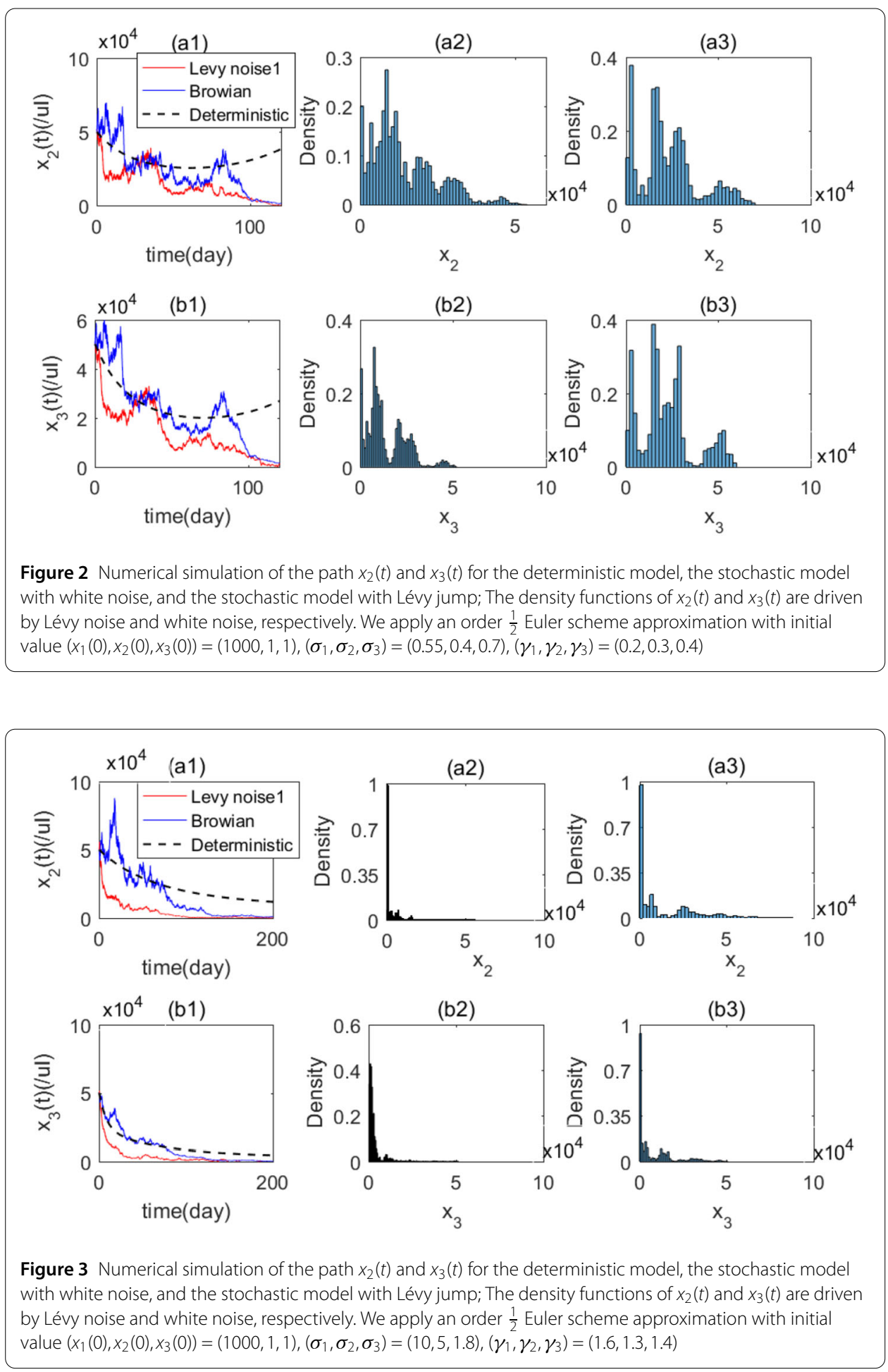

\section{Conclusion}

This paper investigated the dynamics of a stochastic HIV infection model with combined therapy of highly active antiretroviral treatment (HAART) and Lévy jumps. To our knowledge, this part of work has not been done so far. Through theoretical analysis and numerical simulations, we obtained some results about the HIV infection on a cellular level 
driven by Lévy noise. First, we investigated the global existence and unique positive solutions; then, by constructing a suitable stochastic Lyapunov function, we gave the sufficient conditions that $\tilde{R}_{0}<1$, the infected CD4+ cells and virus particles extinct in probability. Then, we adopted a special method to deal with the model, and obtained if $\tilde{R}_{1}>1$ the infected CD4+ cells and virus particles are persistent in mean. By numerical simulations, the theoretical results were verified. We also observed some phenomena that strong perturbation of the environment is beneficial to the extinction of the infected CD4+ cells and virus particles.

Due to the inherent stochastic nature of HIV infection, some interesting topics deserve further discussion, such as considering another common random perturbation regimeswitching that was studied by several authors recently [36, 37]. We will go about these cases subsequently.

\section{Funding}

This work was supported by the Fund of Taiyuan University of Technology (No. 1205-04020203) and the bidding project of Gannan Normal University (16zb02).

\section{Competing interests}

We confirm that none of the authors have any competing interests in the manuscript.

\section{Authors' contributions}

YC carried out the modeling studies, participated in the theoretical analysis, and drafted the manuscript. FZ carried out the simulations of the model. MZ participated in the design of the study and performed the theoretical analysis. All authors read and approved the final manuscript.

\section{Author details}

'School of Mathematical, Taiyuan University of Technology, Taiyuan, China. ${ }^{2}$ Key Laboratory of Jiangxi Province for Numerical Simulation and Emulation Techniques, Gannan Normal University, Gannan, China.

\section{Publisher's Note}

Springer Nature remains neutral with regard to jurisdictional claims in published maps and institutional affiliations.

Received: 29 November 2018 Accepted: 21 April 2019 Published online: 06 August 2019

\section{References}

1. Tan, W.Y., Wu, H.: Stochastic modeling of the dynamics of CD4 ${ }^{+}$T-cell infection by HIV and some Monte Carlo studies. Math. Biosci. 147(2), 173 (1998)

2. Culshaw, R.V., Ruan, S.: A delay-differential equation model of HIV infection of CD4+ T-cells. Math. Biosci. 165, 27-39 (2000)

3. Perelson, A.S., Nelson, P.W.: Mathematical analysis of HIV-1 dynamics in vivo. SIAM Rev. 41(1), 3-44 (1999)

4. Di Mascio, M., Ribeiro, R., Markowitz, M., Ho, D., Perelson, A.: Modelling the long-term control of viraemia in HIV-1 infected patients treated with antiretroviral therapy. Math. Biosci. 188, 47-62 (2004)

5. Bonhoeffer, S., Shaw, G., May, R., Nowak, M.: Virus dynamics and drug therapy. Proc. Natl. Acad. Sci. USA 94, 6971-6976 (1997)

6. Rihan, F.A., Rahman, D.H.A.: Delay differential model for tumour-immune dynamics with HIV infection of CD4 ${ }^{+}$T-cells. Int. J. Comput. Math. 90(3), 594-614 (2013)

7. Yan, M., Xiang, Z:: A delay-differential equation model of HIV infection of $C D 4^{+} T$-cells with cure rate. J. Appl. Math. Comput. 31(1-2), 51-70 (2009)

8. Chen, Z.W.: Impact of intracellular delays and target-cell dynamics on in vivo viral infections. SIAM J. Appl. Math. 70(7/8), 2434-2448 (2010)

9. Nelson, P.W., Murray, J.D., Perelson, A.S.: A model of HIV-1 pathogenesis that includes an intracellular delay. Math. Biosci. 163(2), 201-215 (2000)

10. Bairagi, N., Adak, D.: Dynamics of cytotoxic T-lymphocytes and helper cells in human immunodeficiency virus infection with Hill-type infection rate and sigmoidal CTL expansion. Chaos Solitons Fractals 103, 52-67 (2017)

11. Pawelek, K.A., Liu, S., Pahlevani, F., et al.: A model of HIV-1 infection with two time delays: mathematical analysis and comparison with patient data. Math. Biosci. 235(1), 98-109 (2012)

12. Khalili, S., Armaou, A.: Sensitivity analysis of HIV infection response to treatment via stochastic modeling. Chem. Eng Sci. 63(5), 1330-1341 (2008)

13. Pitchaimani, M., Monica, C.: Global stability analysis of HIV-1 infection model with three time delays. J. Appl. Math. Comput. 48(1-2), 293-319 (2015)

14. Ding, A., Wu, H.: Relationships between antiviral treatment effects and biphasic viral decay rates in modelling HIV dynamics. Math. Biosci. 160, 63-82 (1999) 
15. Martínez, E., Domingo, P., Ribera, E., et al.: Effects of metformin or gemfibrozil on the lipodystrophy of HIV-infected patients receiving protease inhibitors. Antivir. Ther. 8(5), 403 (2003)

16. Sutimin, C.F., Soewono, E., et al.: A model incorporating combined RTIs and PIs therapy during early HIV-1 infection. Math. Biosci. 285, 102 (2017)

17. Tuckwell, H.C., Wan, F.Y.: Nature of equilibria and effects of drug treatments in some simple viral population dynamical models. IMA J. Math. Appl. Med. Biol. 17(4), 311 (2000)

18. Dalal, N., Greenhalgh, D., Mao, X.: A stochastic model for internal HIV dynamics. J. Math. Anal. Appl. 341(2), 1084-1101 (2008)

19. Khalili, S., Armaou, A.: Sensitivity analysis of HIV infection response to treatment via stochastic modeling. Chem. Eng. Sci. 63(5), 1330-1341 (2008)

20. Singh, A., Razooky, B., Cox, C.D., et al.: Transcriptional bursting from the HIV-1 promoter is a significant source of stochastic noise in HIV-1 gene expression. Biophys. J. 98(8), 32-34 (2010)

21. Dalal, N., Greenhalgh, D., Mao, X.: A stochastic model for internal HIV dynamics. J. Math. Anal. Appl. 341(2), 1084-1101 (2008)

22. Huang, Z., Yang, Q., Cao, J.: Complex dynamics in a stochastic internal HIV model. Chaos Solitons Fractals 44(11), 954-963 (2011)

23. Jiang, D., Liu, Q., Shi, N., et al.: Dynamics of a stochastic HIV-1 infection model with logistic growth. Phys. A, Stat. Mech. Appl. 469, 706-717 (2017)

24. Ji, Ch., Jiang, D.: Dyanmical of an HIV-1 infection model with cell-mediated immune response and stochastic perturbation. Int. J. Biomath. 5(5), 103-127 (2012)

25. Wang, Y., Jiang, D., Hayat, T., et al.: A stochastic HIV infection model with T-cell proliferation and CTL immune response. Appl. Math. Comput. 315, 477-493 (2017)

26. Zhang, X., Wang, K.: Stochastic SIR model with jumps. Appl. Math. Lett. 26(8), 867 (2013)

27. Xu, Y., Wang, X.Y., Zhang, H.Q., et al.: Stochastic stability for nonlinear systems driven by Lévy noise. Nonlinear Dyn. $68(1-2), 7-15(2011)$

28. Li, D., Cui, J., Song, G.: Permanence and extinction for a single-species system with jump-diffusion. J. Math. Anal. Appl. 430(1), 438-464 (2015)

29. Zhao, Y., Jiang, D.: The threshold of a stochastic SIS epidemic model with vaccination. Appl. Math. Comput. 243, 718-727 (2014)

30. Zhou, Y., Zhang, W.: Threshold of a stochastic SIR epidemic model with Lévy jumps. Phys. A, Stat. Mech. Appl. 446 204-216 (2016)

31. Meng, L., Bai, C.: Dynamics of a stochastic one-prey two-predator model with Lévy jumps. Appl. Math. Comput. 284(C), 308-321 (2016)

32. Menaldi, J.L.: Applied stochastic control of jump diffusions by B. Øksendal; A. Sulem. SIAM Rev. 47(4), 840-841 (2005)

33. Protter, P., Talay, D.: The Euler scheme for Lévy driven stochastic differential equations. Ann. Probab. 25(1), 393-423 (1997)

34. Fournier, N.: Simulation and approximation of Lévy-driven stochastic differential equations. ESAIM Probab. Stat. 15(1), 233-248 (2011)

35. Vaughan, T.G., Drummond, P.D., Drummond, A.J.: Within-host demographic fluctuations and correlations in early retroviral infection. J. Theor. Biol. 295(2), 86-99 (2012)

36. Meng, L., Xin, H., Yu, J.: Dynamics of a stochastic regime-switching predator-prey model with harvesting and distributed delays. Nonlinear Anal. Hybrid Syst. 28, 87-104 (2018)

37. Meng, L., Du, C., Deng, M.: Persistence and extinction of a modified Leslie-Gower Holling-type II stochastic predator-prey model with impulsive toxicant input in polluted environments. Nonlinear Anal. Hybrid Syst. 27, 177-190 (2018)

\section{Submit your manuscript to a SpringerOpen ${ }^{\circ}$ journal and benefit from:}

- Convenient online submission

- Rigorous peer review

- Open access: articles freely available online

- High visibility within the field

- Retaining the copyright to your article

Submit your next manuscript at $\gg$ springeropen.com 